\title{
Vulvovaginal lichen planus: A case report
}

\section{Singh Th. Nandakishore, Yaku Kago, Linda Kongbam, Romita Bachaspatimayum}

\author{
Department of Dermatology, Venereology and Leprosy, RIMS, Imphal, Manipur, India
}

Corresponding author: Yaku Kago, MD, E-mail: aku.kago00@gmail.com

\begin{abstract}
Genital lichen planus (LP) forms a small but significant proportion of non-venereal genital dermatoses. Lesions of vulvovaginal LP are often asymptomatic and may be overlooked on self-examination. Clinical features are very similar to lichen sclerosus which is frequently seen in children whereas mucosal vulval LP commonly affects adult females. Histopathological examination of the lesion is an important diagnostic tool for its early diagnosis as progressive disease poses a risk of scarring. We report a case of vulvovaginal LP in a 56-year-old postmenopausal female presenting with hypopigmented patches on vulva for 10 years.
\end{abstract}

Key words: Lichen planus; Vulvovaginal; Histopathology; Treatment

\section{INTRODUCTION}

Lichen planus (LP) is a chronic inflammatory dermatosis affecting the skin, mucous membranes, and nails. Genital LP forms a small but significant proportion of non-venereal genital dermatoses [1], in $25 \%$ of the cases of with lichen planus, male genitalia are involved especially the glans penis [2]. The exact incidence of genital LP in women is not known as the lesions are often overlooked on self-examination and occasionally lesions may be asymptomatic. The clinical forms of LP on the female genitalia are mainly erosive, papulosquamous and rarely hypertrophic [1].

\section{CASE REPORT}

A 56-year-old married postmenopausal female presented with pruritus, erythema of genital region with whitish vaginal discharge on and off for 10 years. There was no history of soreness, burning sensation, pain, dyspareunia, contact or post coital bleeding. Patient had no history of autoimmune disease, diabetes, hypertension or gynecological disorder. The patient had not received any treatment. Family history was not significant.
Examination showed erythema over the vulvar and vaginal mucosa with whitish discharge seen at vaginal introitus (Fig. 1) and few ill-defined hypopigmented patches on the labia minora. No scarring was seen in genital mucosa and the hair bearing areas of the genitals (mons pubis, inner thighs) were spared. Oral mucosa, skin and nails were normal. General physical examination was unremarkable.

Histopathological examination (HPE) revealed band like lymphocytic infiltration of dermo-epidermal junction with focal basal cell vacuolar degeneration and presence of Civatte bodies (Fig. 2). Potassium hydroxide examination of vaginal discharge showed Candida spp. Post prandial blood sugar level was slightly elevated $(159 \mathrm{mg} / \mathrm{dl})$. Serological tests for HIV, hepatitis $\mathrm{B}$ and $\mathrm{C}$ were negative. All other routine hematological and biochemical tests were normal.

Patient was started on topical and intralesional steroid along with tablet flucanozole and topical antifungal for candidiasis. Follow up was done 3 weekly and there was significant reduction in pruritus, erythema and vaginal discharge (Fig. 3). After receiving 3 doses of intralesional steroid, she was lost to follow up.

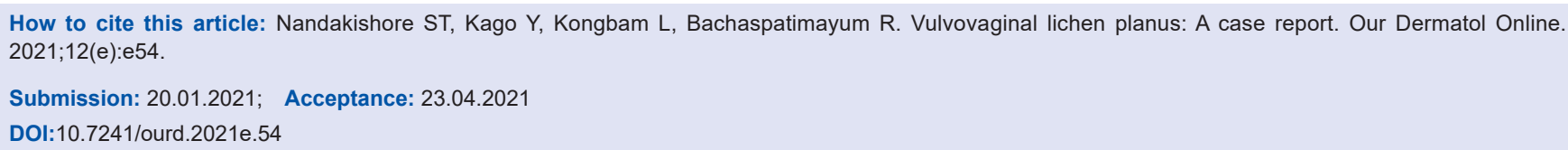




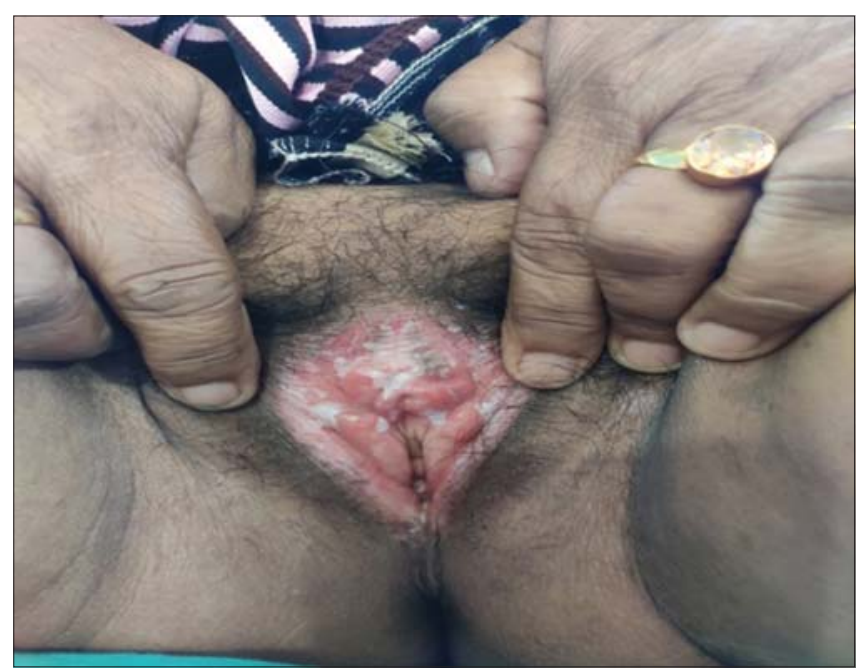

Figure 1: Erythema over the vulvar and vaginal mucosa with whitish discharge seen at vaginal introitus. Few ill-defined hypopigmented patches on the labia minora.

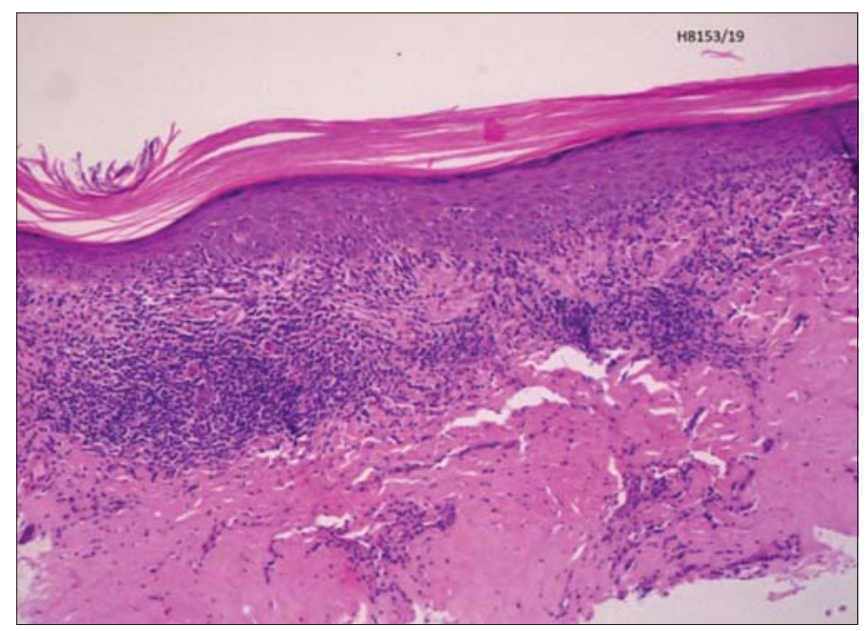

Figure 2: Histopathological examination showing band like lymphocytic infiltration of dermo-epidermal junction with focal basal cell vacuolar degeneration and presence of Civatte bodies (H\&E, 40x).

\section{DISCUSSION}

Genital dermatoses are not easy to diagnose as their morphology is modified by the special environment of the genital area which produces heat, friction and occlusion [3].

The classical cutaneous lesions of LP are rare in the genital region [1] and in females; it typically involves the inner aspects of the labia minora showing a glazed erythema that may easily bleed on touch. Papulosquamous LP presents as violaceus papules or plaques with overlying white reticulations (Wickham's striae). Hypertrophic LP presents as hyperkeratotic lesions, which can mimic squamous cell carcinoma [4].

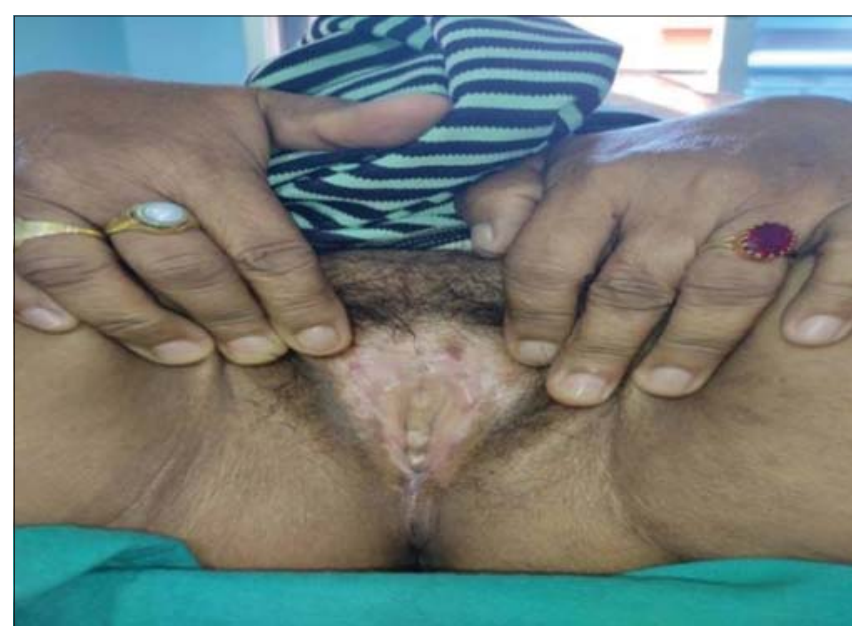

Figure 3: Follow up at 9 weeks, significant reduction in erythema and vaginal discharge.

The classical histopathological findings in both cutaneous and genital lichen planus include hyperkeratosis, wedgeshaped hypergranulosis and saw-toothed acanthosis with a band-like lymphohistiocytic infiltrate obscuring the dermo-epidermal junction. The basal layer shows hydropic degeneration with the presence of apoptotic bodies [1]. Various clinical forms of vulvovaginal LP have different clinical course and complications. The papulosquamous and hypertrophic variants often respond to therapy and do not cause scarring. However, the erosive variant is challenging to treat and can cause scarring and complete remission unfortunately occur in only a small percentage of erosive LP [1].

Progressive disease can lead to scarring of vulva, resorption of labia minora and even shortening of the vagina which, in turn, hinders the sexual activity of the patient [4].

Thus, regular follow-up with prompt biopsy of any atypical lesion such as a persistent non-healing erosion/ ulcer or whitish thickened areas should be done [1]

The treatment outcome varies with the morphological presentation of the disease and topical steroids form the mainstay of treatment but vaginal application carries a risk of iatrogenic Cushing's over long term due to increased absorption owing to thin epithelium and the effect of occlusion. ${ }^{1}$ Topical calcineurin inhibitors have demonstrated improvement in symptoms in cases refractory to topical corticosteroids [4].

Systemic therapy is initiated when topical treatment fails and systemic steroids are possibly the most effective agents showing consistent efficacy [1]. Other systemic immunosuppressive agents used are mycophenolate 
mofetil, methotrexate or azathioprine. Metronidazole and rituximab have also been tried in mucosal LP. Photodynamic therapy (PDT) is the most recently described treatment and was found to have an equivalent efficacy to clobetasol ointment in a randomized controlled trial. Adhesiolysis may be needed for severe cases [4].

\section{CONCLUSION}

Genital LP lesions are often asymptomatic and may be overlooked by women on self- examination. As the clinical presentation is very similar to lichen sclerosus, HPE of the lesion becomes an important diagnostic tool to differentiate them. Progressive disease poses a risk of scarring of the genital region and development of neoplasms. Hence, early recognition and treatment with regular follow up is necessary.

\section{Consent}

The examination of the patient was conducted according to the principles of the Declaration of Helsinki.
The authors certify that they have obtained all appropriate patient consent forms, in which the patients gave their consent for images and other clinical information to be included in the journal. The patients understand that their names and initials will not be published and due effort will be made to conceal their identity, but that anonymity cannot be guaranteed.

\section{REFERENCES}

1. Khurana A, Tandon S, Marfatia YS, Madnani N. Genital lichen planus: An under recognized entity. Indian J Sex Transm Dis. 2019;40:105-12.

2. Zaouak A, Bouhajja L, Hammami H, Fenniche S. Penile annular lichen planus. Our Dermatol Online. 2020;11:218-9.

3. Puri N, Puri P. A study on non venereal genital dermatoses in north India. Our Dermatol Online. 2013;4:304-7.

4. Fruchter R, Melnick L, Pomeranz MK. Lichenoid vulvar disease: A review. Int J Women Dermatol. 2017;3:58-64.

Copyright by Singh Th. Nandakishore, et al. This is an open-access article distributed under the terms of the Creative Commons Attribution License, which permits unrestricted use, distribution, and reproduction in any medium, provided the original author and source are credited.

Source of Support: Nil, Conflict of Interest: None declared. 